\title{
Striped Spin Liquid Crystal Ground State Instability of Kagome Antiferromagnets
}

\author{
Bryan K. Clark, ${ }^{1,2}$ Jesse M. Kinder, ${ }^{3,4}$ Eric Neuscamman, ${ }^{5,4}$ Garnet Kin-Lic Chan, ${ }^{6,4}$ and Michael J. Lawler ${ }^{7,8, *}$ \\ ${ }^{1}$ Station Q, Microsoft Research, Santa Barbara, California 93106, USA \\ ${ }^{2}$ Princeton University and Princeton Center for Theoretical Science, Department of Physics, Princeton, New Jersey 08544, USA \\ ${ }^{3}$ Case Western Reserve University, Department of Physics, Cleveland, Ohio 44106, USA \\ ${ }^{4}$ Cornell University, Department of Chemistry, Ithaca, New York 14853, USA \\ ${ }^{5}$ University of California Berkeley, Department of Chemistry, Berkeley, California 94720, USA \\ ${ }^{6}$ Princeton University, Department of Chemistry, Princeton, New Jersey 08544, USA \\ ${ }^{7}$ Department of Physics, Binghamton University, Binghamton, New York 13902-6000, USA \\ ${ }^{8}$ Department of Physics, Cornell University, Ithaca, New York 14853, USA
}

(Received 5 November 2012; published 1 November 2013)

\begin{abstract}
The Dirac spin liquid ground state of the spin 1/2 Heisenberg kagome antiferromagnet has potential instabilities. This has been suggested as the reason why it does not emerge as the ground state in largescale numerical calculations. However, previous attempts to observe these instabilities have failed. We report on the discovery of a projected BCS state with lower energy than the projected Dirac spin liquid state which provides new insight into the stability of the ground state of the kagome antiferromagnet. The new state has three remarkable features. First, it breaks spatial symmetry in an unusual way that may leave spinons deconfined along one direction. Second, it breaks the $U(1)$ gauge symmetry down to $Z_{2}$. Third, it has the spatial symmetry of a previously proposed "monopole" suggesting that it is an instability of the Dirac spin liquid. The state described herein also shares a remarkable similarity to the distortion of the kagome lattice observed at low $\mathrm{Zn}$ concentrations in $\mathrm{Zn}$-paratacamite and in recently grown single crystals of volborthite suggesting it may already be realized in these materials.
\end{abstract}

The ground state of the spin $1 / 2$ kagome antiferromagnet is likely a very novel form of magnetism; proposals include magnetic ordering in one of several spin patterns [1], a valence bond crystal with a 36 site unit cell [2] or a 12 site unit cell [3], a chiral spin liquid [2,4], several kinds of gapped spin liquids [5,6], and a gapless algebraic spin liquid $[7,8]$. Many of these states can be represented by a projection of a Slater determinant or BCS wave function onto the insulating one fermion per site subspace-the socalled fermion slave particle method. One ground state that is particularly competitive and novel is the $U(1)$ Dirac spin liquid (DSL), a gapless algebraic spin liquid. The DSL is an isotropic spin liquid governed at low energies by quantum electrodynamics in two spatial dimensions. Its spin carrying excitations are gapless Dirac fermions with a linear dispersion relation around two degenerate points in the band structure similar to the electronic structure of graphene. Its singlet excitations are characterized by gapless photons arising from the emergent $U(1)$ gauge symmetry of the mean-field Hamiltonian.

Many other candidate ground states of the kagome antiferromagnet can be written as instabilities of the DSL; these have been cataloged in Ref. [9]. Despite the existence of these potential instabilities, variational studies that include the DSL report it to be remarkably stable [8-12].

Recently, large scale density matrix renormalization group (DMRG) calculations $[3,13,14]$ have produced strong numerical evidence that the true ground state of the kagome antiferromagnet is a $Z_{2}$ spin liquid, where the name $Z_{2}$ refers to the gapping out of the $U(1)$ gauge boson much like the Meissner effect in a superconductor. In particular, the best variational DMRG state has a gap to all excitations and the topological entanglement entropy expected of a $Z_{2}$ spin liquid.

Perhaps the simplest explanation of the DMRG results, as proposed in Ref. [6], is that spin carrying Dirac fermions pair up to form a superfluid. These authors catalog 20 possible such states and give explicit instructions for constructing 14 of them. However, variational studies [12] have failed to find any of these states with a lower energy than the DSL.

In this Letter, we revisit the projected BCS (PBCS) variational wave function problem on the kagome lattice and the stability of the DSL. Building on Refs. $[15,16]$, we explore the entire set of time-reversal invariant projected BCS wave functions by optimizing the symmetric pairing matrix that characterizes these states. This wave function can describe $U(1)$ and $Z_{2}$ spin liquids, general valence bond solids, and many other potential instabilities of the DSL. The most important results of our study are the following:

(1) There are projected BCS wave functions with lower energy than the DSL. Figure 1 shows the spin correlations of a wave function with lower energy than the DSL on a 48-site lattice. The optimal state breaks lattice symmetry and doubles the unit cell; however, it preserves $C_{2}$ rotational symmetry about several lattice points, which gives it a one-dimensional character. We refer to this new state as a 


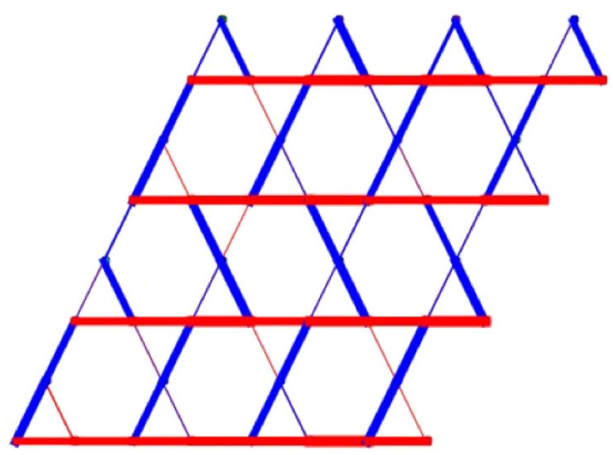

FIG. 1 (color online). The nearest neighbor $\left\langle\Psi\left|\vec{S}_{i} \cdot \vec{S}_{j}\right| \Psi\right\rangle$ correlations for the lowest energy projected mean field state. Red means stronger $\left\langle\Psi\left|\vec{S}_{i} \cdot \vec{S}_{j}\right| \Psi\right\rangle$, blue means weaker, and the linewidth measures the magnitude of deviations from the mean. On average, the red bonds are $0.8 \%$ lower energy and the blue bonds are $1.4 \%$ higher energy than the mean.

striped spin liquid crystal (SSLC). This state fundamentally challenges the typical approach for searching for spin liquids, a search focused on isotropic states, for it shares many features of these states even though it breaks crystal symmetries.

(2) A symmetry analysis suggests that these states of lower energy are instabilities of the DSL. $S U(2)$ flux patterns can distinguish among different classes of spin liquids. We find that the SSLC and the DSL have nearly identical flux patterns. However, our state requires pairing in the projected BCS form: we find no states with lower energy than the DSL if the optimization is restricted to states with $U(1)$ gauge symmetry. We conjecture that broken symmetry and lower energy result from a proliferation of spin-singlet, nodal-triplet $w$ monopoles, an instability of the DSL described in Ref. [8].

(3) There is reason to believe the SSLC appears in real materials. The pattern of spin correlation shown in Fig. 1 has been observed in the structural distortion pattern of kagome layers of single crystals of volborthite and in samples of Zn-paratacamite with $\mathrm{Zn}$ concentrations less than one-third $[17,18]$. These materials are ideal candidates for further understanding the properties of this exotic state.

Method.-Our objective is to find the lowest energy state of the nearest neighbor spin $1 / 2$ kagome antiferromagnet within the set of Gutzwiller projected BCS wave functions:

$$
|\Psi\rangle=\hat{\mathcal{P}}_{N}\left|\Psi_{0}\right\rangle
$$

$\left|\Psi_{0}\right\rangle$ is a BCS wave function. For an electron configuration on the lattice given by $\vec{R}=\left(\vec{r}_{1}, \vec{r}_{2}, \ldots, \vec{r}_{N}\right)$, the amplitude is a determinant $\Psi_{0}(\vec{R})=\operatorname{det} M(\vec{R})$. The elements of $M$ are derived from pairing amplitudes: $M_{i j}(\vec{R})=\phi\left(\vec{r}_{i}, \vec{r}_{j}\right) . \hat{\mathcal{P}}_{N}$ projects out configurations which do not have one electron per site. This class of states includes the DSL and all of the states cataloged in Ref. [6], but is not restricted to these. We minimize the energy of the Heisenberg spin
Hamiltonian on the kagome lattice, $H=\sum_{\langle i j\rangle} \vec{S}_{i} \cdot \vec{S}_{j}$, by varying all $N(N-1) / 2$ elements of the pairing function $\phi\left(\vec{r}_{i}, \vec{r}_{j}\right)$ using the methods described in Refs. $[15,16]$.

To explore this energy landscape, we used the projective symmetry group classification $[6,8,9,19]$ to start the optimization in many qualitatively different spin liquid states. We used the mean field parameter defined in Ref. [6] to derive a pairing matrix as described in the Supplemental Material, S-I and S-II [20]. This allowed us to start our optimization in 14 different classes of spin liquid states.

Figure 2 shows typical optimization traces of the energies for each spin liquid state on the $4 \times 4$ lattice ( 48 sites), labeled following Table II of Ref. [6].

On a 48-site lattice, the DSL gives an energy per site of $-0.42938 \pm 4 \times 10^{-5}$. Many states in our study have lower energies; our best variational state has an energy per site of $-0.43052 \pm 5 \times 10^{-5}$. Thus, the DSL is not the most stable projected mean field state. We have found lower energy states on clusters of up to 192 sites with a trend towards the same spatial symmetry breaking as shown in the Supplemental Material S-III [20,21].

This is particularly surprising in light of previous studies that find the Dirac spin liquid to be stable against many instabilities $[7-9,12,22]$. These studies failed to find the SSLC because they were restricted to highly symmetric, short-range mean-field Hamiltonians. We avoid this limitation by optimizing all elements of the pairing matrix. This effectively introduces arbitrary long-range hopping and pairing and therefore allows breaking arbitrary spatial symmetries of the mean-field Hamiltonian.

Symmetry.-Let us then focus on the nature of our lowest energy state. Most strikingly, we find that the state breaks

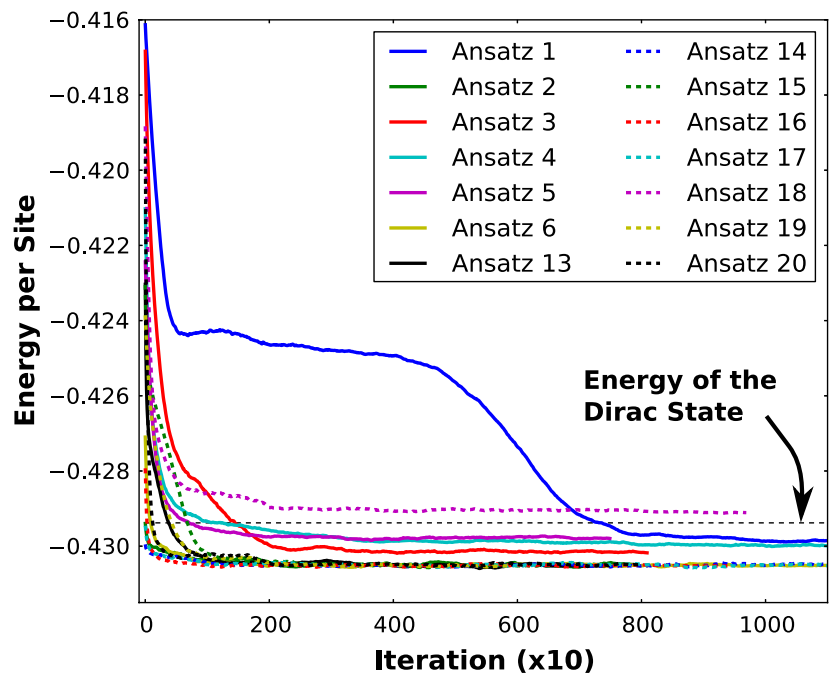

FIG. 2 (color online). (a) Typical stochastic optimization traces starting from the 14 distinct PSG ansatz (ansatz 1-6 and 13-20 in Table II of Ref. [6]), and optimizing the pairing function $\phi$. Highlighted dashed grey line shows the energy of the Dirac state. Many traces end at an energy below this line. 
orientational and translation symmetry with a doubling of the unit cell. Figure 1 shows deviations of the nearest neighbor spin-spin correlation function from its mean (on average, the blue bonds are $0.8 \%$ higher energy and the red bonds are $1.4 \%$ lower energy than the mean). In contrast, the DSL has slightly anisotropic spin correlations due to the use of twisted boundary conditions on finite clusters (as shown in Supplemental Material S-IV [20]) but no doubling of the unit cell. The pairing matrix that projects to our lowest energy state is shown in Supplemental Material S-V [20] and has different symmetry but includes unphysical aspects of the unprojected wave function.

We have checked that the broken symmetry state of Fig. 1 has no nearby isotropic spin liquid. Starting near our lowest energy state, we searched for a nearby isotropic spin liquid by stochastically moving toward states which had smaller deviations in their unprojected spin correlation function $\left\langle\vec{S}_{i} \cdot \vec{S}_{j}\right\rangle$. This procedure recovered a symmetric state with the same energy as the DSL. This suggests there are no other isotropic spin liquids "close to" our state or the DSL in the space of projected BCS wave functions and that symmetry breaking in addition to longer range hopping and pairing terms are important for this state.

Having found a broken symmetry state, we would like to understand its relation to known symmetric spin liquids. The anomalous density matrix (ADM) of the unprojected wave function is a useful analytic tool [23]. It can be used to calculate an $S U(2)$ flux through loops of bonds in the lattice, and the flux pattern can then be used to distinguish among the different spin liquids of Ref. [6]. The fluxes of our SSLC lets us deduce which spin liquid our state resembles. The ADM is given by

$$
\rho_{i j}=\left(\begin{array}{cc}
-A_{i j}^{*} & B_{i j} \\
B_{i j}^{*} & A_{i j}
\end{array}\right),
$$

where

$$
A_{i j}=\frac{\left\langle\Psi_{0}\left|f_{i \downarrow}^{\dagger} f_{j \downarrow}\right| \Psi_{0}\right\rangle}{\left\langle\Psi_{0} \mid \Psi_{0}\right\rangle}, \quad B_{i j}=\frac{\left\langle\Psi_{0}\left|f_{i \uparrow} f_{j \downarrow}\right| \Psi_{0}\right\rangle}{\left\langle\Psi_{0} \mid \Psi_{0}\right\rangle},
$$

and transforms under an $S U(2)$ gauge transformation, $\Psi \rightarrow \mathbf{G} \cdot \Psi$, like $\rho_{i j} \rightarrow \mathbf{G}_{i} \cdot \rho_{i j} \cdot \mathbf{G}_{j}^{\dagger}$ - exactly like the mean fields in the corresponding slave particle theory (see Ref. [24] or the Supplemental Material S-I [20]). We can therefore use this matrix to study the projective symmetry properties of the optimized unprojected state $\left|\Psi_{0}\right\rangle$.

We define the $S U(2)$ matrix [see Ref. [19], Eq. (13)]

$$
\mathbf{W}_{i j}=-i \rho_{i j} / \sqrt{-\operatorname{det} \rho_{i j}},
$$

that is an analog of the $U(1)$ phase variable $e^{i a_{i j}}$ of ordinary electricity and magnetism on a lattice. The $S U(2)$ flux matrix through any loop on the lattice is then the product of $\vec{W}_{i j}$ around a loop

$$
\Phi_{i j k \ldots l}=i^{N_{\text {loop }}} \mathbf{W}_{i j} \cdot \mathbf{W}_{j k} \cdot \ldots \cdot \mathbf{W}_{l i}=e^{i(\theta / 2) \hat{n} \cdot \boldsymbol{\tau}},
$$

where $N_{\text {loop }}$ is the number of bonds $i j$ that form the loop. This product is not gauge invariant; however, the trace is gauge invariant and the angle $\theta$ derived from it can be used to distinguish different phases.

Natural loops to characterize the state $\left|\Psi_{0}\right\rangle$ are nearestneighbor bow ties (a "figure 8 " enclosing two neighboring triangles) and hexagons. (The trace of the flux through odd-site loops vanishes by time-reversal symmetry.) These loops allow us to determine which of the $U(1)$ spin liquid states is most closely related to ours. The DSL has an $S U$ (2) flux angle of $2 \pi$ for each hexagon and 0 for each bow tie. The SSLC flux angles are nearly identical:

$$
\begin{aligned}
\left\langle\theta_{\text {hex }}\right\rangle & =(1.994 \pm 0.003) \pi, \\
\left\langle\theta_{\text {bow }}\right\rangle & =(0.010 \pm 0.007) \pi,
\end{aligned}
$$

where the error estimate is the standard deviation and $\langle\ldots\rangle$ denotes the average value of the flux over all hexagons or bow ties, respectively. This proximity to the DSL in energy and flux angles leads us to conclude that the striped spin liquid crystal is an instability of the $U(1)$ Dirac spin liquid.

Having established that our newly discovered state is very close to the Dirac state, we now turn to its symmetry breaking properties. Reference [9] contains a catalog of space group representations of the kagome lattice which require at most a quadrupling of the unit cell. Those with the same period as the kagome lattice are labeled as $A_{1}, A_{2}$, $B_{1}, B_{2}, E_{1}$, and $E_{2}$. Those that double or quadruple the unit cell are labeled $F_{1}, F_{2}, F_{3}$, and $F_{4}$. The focus of Ref. [9] was on the $F_{1}$ representation for the "Hastings valence bond crystal" states associated with the generation of mass of the Dirac fermions. However, the bond amplitudes in Fig. 1 are dominated by the $E_{2}$ and $F_{2}$ representations in Figs. 3(a) and 3(b).

This is clearly seen in Figs. 3(c) and 3(d). There, we plot energy as a function of asymmetry in the uniform and $F_{2}$ components, respectively. In both cases, we subtract out the $E_{2}$ component to remove the effect of twisted boundary conditions. This gives us

$$
\mathcal{O}_{\text {uniform }}=\sum_{\langle i j\rangle}\left\langle\Psi\left|\vec{S}_{i} \cdot \vec{S}_{j}\right| \Psi\right\rangle-E_{2 i j}^{1}\left\langle\Psi\left|\vec{S}_{i} \cdot \vec{S}_{j}\right| \Psi\right\rangle,
$$

and

$$
\mathcal{O}_{F_{2}}=\sum_{\langle i j\rangle} F_{2 i j}^{1}\left\langle\Psi\left|\vec{S}_{i} \cdot \vec{S}_{j}\right| \Psi\right\rangle
$$

where $E_{2 i j}^{1}$ and $F_{2 i j}^{1}$ are defined in Figs. 3(a) and 3(b).

Note that any state which breaks $F_{2}$ symmetry generically also breaks $E_{2}$ symmetry. Therefore, the symmetry breaking of Fig. 1 can be entirely regarded as a breaking of the $F_{2}$ symmetry. Since no $F_{1}$ representation is present, and this pattern is associated with mass terms [9], the spatial symmetry breaking alone does not generate a gap. 


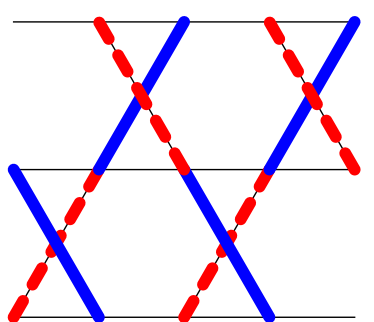

(a)

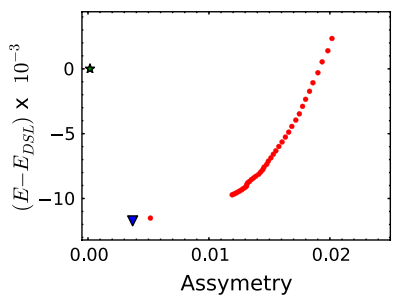

(c)

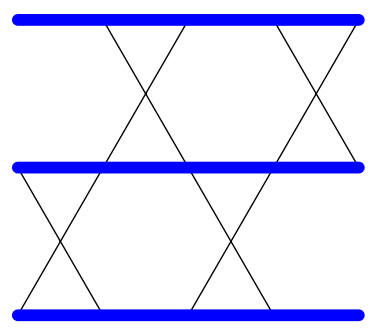

(b)

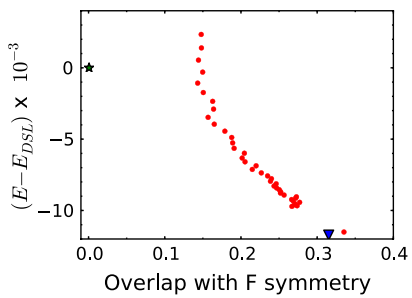

(d)
FIG. 3 (color online). Spatial symmetry analysis of our state. (a) one orientation $F_{2 i j}^{1}$ of the $F_{2}$ pattern. On the blue solid bonds, $F_{2 i j}^{1}=\sqrt{3 / 2 N_{b}}$; on the red dashed bonds, $F_{2 i j}^{1}=$ $-\sqrt{3 / 2 N_{b}}$; on the thin black bonds, $F_{2 i j}^{1}=0$ (here, $N_{b}$ the number of nearest neighbor bonds). (b) one component $E_{2 i j}^{1}$ of the $E_{2}$ pattern. On the thick blue bonds, $E_{2 i j}^{1}=\sqrt{2 / N_{b}}$; on the thin black bonds, $E_{2 i j}^{1}=-1 / \sqrt{2 N_{b}}$. These patterns clearly arise in Fig. 1. (c) and (d) correlation between the energy of different states and the amount of asymmetry [(c) as defined by Eq. (7); (d) as defined by Eq. (8)]. Red circles correspond to states generated during part of an optimization, the green star to the Dirac spin liquid, and the blue triangle to our most optimized state. For energies below the Dirac spin liquid, improved energy is correlated with decreased total asymmetry but increased strength of the $F_{2}$ pattern.

Among instabilities of the Dirac fixed point identified in Ref. [9], the only time reversal symmetric alternative to the Hastings states is the spin-singlet, nodal-triplet $w$ monopole. In Supplemental Material S-VI [20], we show that the six dimensional $w$-monopole operator transforms under the three dimensional representations of both $F_{1}$ and $F_{2}$. This remarkable coincidence leads us to conjecture that the $w$ monopole is responsible for the instability of the Dirac state that leads to Fig. 1. A proliferation of this monopole should lead to confinement, but neither magnetic ordering nor dimerization is observed. The only natural candidate for confinement is a pinning of the spinon to the "rails" with the strongest $\left\langle\vec{S}_{i} \cdot \vec{S}_{j}\right\rangle$ bonds.

In addition to breaking the spatial symmetries of the lattice, the physical system also breaks the $U(1)$ symmetry of the Dirac state down to a $Z_{2}$ symmetry. To study this symmetry breaking, we optimized a series of wave functions whose pairing matrices exclude the possibility of pairing [i.e., $B_{i j}=0$ in Eq. (2)]. Starting from the $U(1)$ DSL, we multiplied each element of its pairing matrix by a random scale factor $(1+r)$, where $r$ was drawn from a normal distribution with zero mean and standard deviation $X$. Figure 2(b) of Supplemental Material S-IV [20] shows 12 optimization runs with $X \in\{0.1,0.2,0.33\}$. The variation in initial energy was quite large-as high as -0.375 per site. However, all simulations converge to the energy of the Dirac spin liquid.

From these results, we conclude that the Dirac state lies at the bottom of a deep and wide region in orbital space and that the pairing of spinons is necessary to produce the state shown in Fig. 1.

Discussion.-Based on our results, it seems very likely that the Dirac fixed point is unstable to the formation of a striped spin-liquid crystal phase. This conclusion rests on two assumptions: (1) the symmetry breaking we observe is not a finite size effect; (2) that fluctuations beyond those captured by the projected wave function do not restore the symmetry. There is indirect evidence that the former assumption is valid. DMRG and exact diagonalization suggest that a 48-site cluster is large enough to capture the qualitative physics of the system. The latter assumption is more difficult to justify but one way would be to construct the low energy effective field theory which corresponds to our striped spin liquid crystal. This can be done by performing a PSG analysis on the relevant lower symmetry subgroup of the kagome lattice and use it to search for a mean field Hamiltonian whose BCS wave function projects to our state. (Since projection is a many-to-one mapping, we cannot deduce the PSG of our state directly.) Such an analysis would allow one to extrapolate to the thermodynamic limit with fixed number of variational parameters and provide a starting point for studying fluctuations about this phase.

Though our variational state may not describe the ideal Heisenberg model, as DMRG calculations suggest a $Z_{2}$ state, perturbations to this model may stabilize it. From an experimental viewpoint, there are a number of real kagome lattice materials which have perturbations which would promote the SSLC state. One promising class of materials is the $\mathrm{Zn}$-paratacamite family parametrized by $\mathrm{Zn}$ doping concentration $x$ with $x<1 / 3$. Unlike the structurally perfect kagome lattice of the $x=1$ Herbertsmithite member of the family, compounds with $x<1 / 3$, including clinoatacamite at $x=0$, break crystal symmetries and have distorted kagome layers [17] with precisely the distortion expected from the symmetry breaking of our state. Single crystals of volborthite show the same distortion [18]. Our results, therefore, motivate the study of single crystals of these materials and suggests that above their magnetic order transition temperature, they could have deconfined spinons propagating along the "rails" that could conduct heat much better in this direction than across the rails.

The most remarkable implication of our results is the discovery of a spin liquid crystal as a low energy phase of an ordinary spin Hamiltonian. This suggests that such phases could be competitive in many other spin liquid candidate systems. One explanation for this competition 
is the role of projection in slave-particle theory. Since any dimer state is the exact ground state at the mean field level [25], projection effectively introduces quantum fluctuations that melt this "crystal" into liquid crystalline phases. Such a picture has implications for the DMRG calculations on the kagome lattice and more generally for the search for exotic phases in magnetic insulators. In DMRG, small perturbations to the Hamiltonian (boundary conditions, pinning fields, etc.) can bias the algorithm toward states with a particular symmetry when there are competing phases [3]. Exploring the class of perturbations that stabilize the symmetry breaking of Fig. 1 in DMRG would provide further insight into its origins and properties. It is even possible that the DMRG ground state on a long cylinder is an instability of the striped spin liquid crystal: a nematic spin liquid crystal that breaks a lattice point group symmetry like that reported in a recent study of a triangular lattice antiferromagnet with ring exchange [26].

We acknowledge useful discussions with Matthew Fisher, Michael Hermele, Zenji Hiroi, David Huse, and Nandini Trivedi. This work used the Extreme Science and Engineering Discovery Environment (XSEDE), which is supported by National Science Foundation Grant No. OCI1053575 and the High Performance Computing Cluster at Case Western Reserve University. B. K. C and J. M. K. contributed equally to this work.

*mlawler@binghamton.edu

[1] A. Chubukov, Phys. Rev. Lett. 69, 832 (1992).

[2] J. B. Marston and C. Zeng, J. Appl. Phys. 69, 5962 (1991).

[3] S. Yan, D. A. Huse, and S. R. White, Science 332, 1173 (2011).

[4] L. Messio, B. Bernu, and C. Lhuillier, Phys. Rev. Lett. 108, 207204 (2012).

[5] S. Sachdev, Phys. Rev. B 45, 12377 (1992).

[6] Y.-M. Lu, Y. Ran, and P. A. Lee, Phys. Rev. B 83, 224413 (2011).

[7] M. B. Hastings, Phys. Rev. B 63, 014413 (2000).

[8] Y. Ran, M. Hermele, P. A. Lee, and X.-G. Wen, Phys. Rev. Lett. 98, 117205 (2007).
[9] M. Hermele, Y. Ran, P. A. Lee, and X.-G. Wen, Phys. Rev. B 77, 224413 (2008).

[10] M. Hermele, T. Senthil, and M. P. A. Fisher, Phys. Rev. B 72, 104404 (2005).

[11] F. S. Nogueira and H. Kleinert, Phys. Rev. Lett. 95, 176406 (2005).

[12] Y. Iqbal, F. Becca, and D. Poilblanc, Phys. Rev. B 83, 100404 (2011).

[13] S. Depenbrock, I. P. McCulloch, and U. Schollwock, Phys. Rev. Lett. 109, 067201 (2012).

[14] H.-C. Jiang, Z. Wang, and L. Balents, Nat. Phys. 8, 902 (2012).

[15] E. Neuscamman, C. J. Umrigar, and Garnet Kin-Lic Chan, Phys. Rev. B 85, 045103 (2012).

[16] B. K. Clark, D. A. Abanin, and S. L. Sondhi, Phys. Rev. Lett. 107, 087204 (2011).

[17] S.-H. Lee, H. Kikuchi, Y. Qiu, B. Lake, Q. Huang, K. Habicht, and K. Kiefer, Nat. Mater. 6, 853 (2007).

[18] H. Yoshida, J.-I. Yamaura, M. Isobe, Y. Okamoto, G. J. Nilsen, and Z. Hiroi, Nat. Commun. 3, 860 (2012).

[19] X.-G. Wen, Phys. Rev. B 65, 165113 (2002).

[20] See Supplemental Material at http://link.aps.org/ supplemental/10.1103/PhysRevLett.111.187205 for background information on the mean field theory and trial wave functions, additional numerical results, and a group theoretical analysis of the $w$-monopole operator.

[21] Optimization and analysis of the wave functions on the larger 192-site cluster require considerably more time and computational resources than the 48-site cluster discussed here. A full discussion of finite size effects will be presented elsewhere.

[22] O. Ma and J. B. Marston, Phys. Rev. Lett. 101, 027204 (2008).

[23] The mean field Hamiltonian cannot be reconstructed from the pairing matrix, but the ADM can. The pairing matrix only contains information about the eigenvectors-not the eigenvalues - of the parent Hamiltonian.

[24] X.-G. Wen, Quantum Field Theory of Many-Body Systems (Oxford University Press, Oxford, U.K., 2004).

[25] D. S. Rokhsar, Phys. Rev. B 42, 2526 (1990).

[26] T. Grover, N. Trivedi, T. Senthil, and P. A. Lee, Phys. Rev. B 81, 245121 (2010). 